ВОЛОДАРСКИЙ В. А., к. т. н., с. н. с., профессор (Красноярский институт железнодорожного транспорта)

\title{
О надежности технических устройств, прошедших ремонт
}

Предложены выражения для определения средней интенсивности отказов и наработки на отказ ремонтируемых технических устройств. Изложены результаты исследований зависимости интенсивности отказов и наработки на отказ от глубины восстановления безотказности, числа и периодичности ремонтов до замень устройств, а также от коэффициента вариации распределений, используемьх для описания отказов технических устройств.

Ключевые слова: интенсивность отказов, наработка на отказ, безотказность, глубина восстановления, ремонт, замена, периодичность.

\section{1. Состояние вопроса}

Известно, что проведение предупредительных замен (ПЗ) и предупредительных ремонтов (ПР) приводит к повышению уровня надежности технических устройств (ТУ). Очевидно, что при ремонте ТУ им обычно возвращается лишь часть исходных надежностных свойств.

Проводить ПЗ и ПР следует только для устройств с постепенными отказами, вызванными процессами износа и старения ТУ. Интенсивность отказов у таких устройств со временем эксплуатации монотонно возрастает. Предупредительные замены и ремонты предотвращают рост интенсивности отказов, обеспечивая необходимый уровень безотказности устройств. Для описания постепенных отказов, как правило, используются распределения с коэффициентом вариации $V<1$ : Вейбулла (с параметром формы $b>1$ ) и гамма (с параметром формы $m>1)$ [1], а также при неполноте исходных данных целесообразно использовать распределение косинуса [2].

Представляется целесообразным эффективность ПЗ и ПР оценивать по улучшению наиболее наглядных показателей безотказности ТУ, таких как интенсивность отказов(ИО) и наработка на отказ (HHO).

Актуальность проблемы имеет два аспекта. Вопервых, необходимо предложить метод учета глубины восстановления безотказности при проведении ремонта ТУ. Во-вторых, установить зависимости значений ИО и ННО от глубины восстановления безотказности технических устройств.

Как в нашей стране, так и за рубежом уделяется определенное внимание учету глубины восстановления надежности при обслуживании технических устройств.

() В.А. Володарский, 2017
Так, в [3] надежность работы системы исследуется при возникновении отказов двух типов. Отказы первого типа устраняются минимальным ремонтом, не изменяющим надежность системы, а отказы второго типа устраняются заменой, полностью обновляющей надежность системы. В [4] при разработке математической модели замены электрооборудования учтена неравноценность отказов, которые разделены на две группы: восстанавливаемые, устраняемые ремонтом, и невосстанавливаемые, устраняемые заменой. В [5] несовершенство обслуживания предлагается оценивать вероятностью того, что плановое воздействие приведет систему в состояние, аналогичное новому состоянию, и вероятностью того, что это воздействие не обновит систему. В [6] также используется вероятность того, что профилактическое обслуживание несовершенно и система при этом отказывает сразу после такого обслуживания. В перечисленных публикациях учитываются только два крайних случая глубины восстановления надежности систем: никакого обновления и полное обновление. Практический же интерес представляют промежуточные значения глубины восстановления надежности между этими крайними случаями.

Естественный процесс старения и износа в [7] учитывается возрастанием числа отказов в каждом последующем межремонтном интервале. В разработанных моделях управления надежностью авиационной техники [8] полнота выполняемых профилактических мероприятий оценивается по изменению интенсивности отказов различных элементов. В работе [9] при проведении профилактического обслуживания введено понятие фактора улучшения, который предложено оценивать экспертным путем. Вопросы учета глубины восстановления надежности рассматривались в перечисленных публикациях только на теоретическом уровне и далеки от практической реализации. Относительно в лучшей степени вопросы оценки 
глубины восстановления надежности решаются при ремонте машин в сельском хозяйстве, где введено понятие коэффициента восстановления ресурса при капитальном ремонте [10].

Для учета глубины восстановления безотказности автором предложено использовать параметр $a>0$, означающий “возраст” ТУ после проведения ремонта [11]. При этом значение параметра $a$ определяется объёмом работ по замене или восстановлению составных частей ТУ при проведении ПР.

Цель статьи - предложить выражения для определения средней интенсивности отказов и наработки на отказ ремонтируемых устройств и провести исследования зависимостей интенсивности отказов и наработки на отказ от глубины восстановления безотказности, от числа и периодичности ремонтов до замены технических устройств, а также от коэффициента вариации распределений, используемых для описания отказов технических устройств.

\section{2. Интенсивность отказов устройств, прошедших} ремонт

Так как распределение наработки на отказ при постепенных отказах не подчиняется экспоненциальному закону, представляется целесообразным использовать среднюю ИО за некоторое заданное время, например, за период ремонта или замены. В [12] рассмотрен случай проведения идеального ремонта, при котором происходит полное восстановление первоначальной безотказности, которое возможно только при замене ТУ на новое. При проведении ПЗ с периодичностью $\tau$ интенсивность постепенных отказов $\lambda(t)$, связанных с износом и старением ТУ, снижается до нуля (см. рис. 1). При этом изменяется распределение наработки на отказ, поскольку зависимость ИО от времени эксплуатации tзаменяется на пилообразную кривую, с размахом от нуля до $\lambda(\tau)$, а затем снова до нуля. Среднее значение ИО $\bar{\lambda}$ (см. пунктир на рис. 1) при проведении ПЗ определяется из выражения

$\bar{\lambda}=\tau^{-1} \int_{0}^{\tau} \lambda(t) d t=-\ln P(\tau) \tau^{-1}$,

где $P(\tau)$ - вероятность безотказной работы при наработке $\tau$.

В общем случае для восстановления безотказности после проведения $n$ ремонтов осуществляется замена ТУ на новое. Характер изменения интенсивности отказов представлен на рис. 2. После проведения ПР с периодичностью $\tau$ ИО снижается до значения $\lambda(a)$, а после проведения ПЗ с периодичностью $\tau_{p}-$ до нуля.
ИО в момент проведения ПР и ПЗ составляет $\lambda(\tau+a)$. Таким образом, кривая интенсивности отказов заменяется на пилообразную с размахом от $\lambda(a)$ до $\lambda(\tau+a)$ и затем до $\lambda(a)$ при проведении ПР и от $\lambda(\tau+a)$ до нуля при проведении ПЗ. С уменьшением периодичности ПР максимумы пилообразной кривой ИО приближаются к прямой $\lambda(a)$.

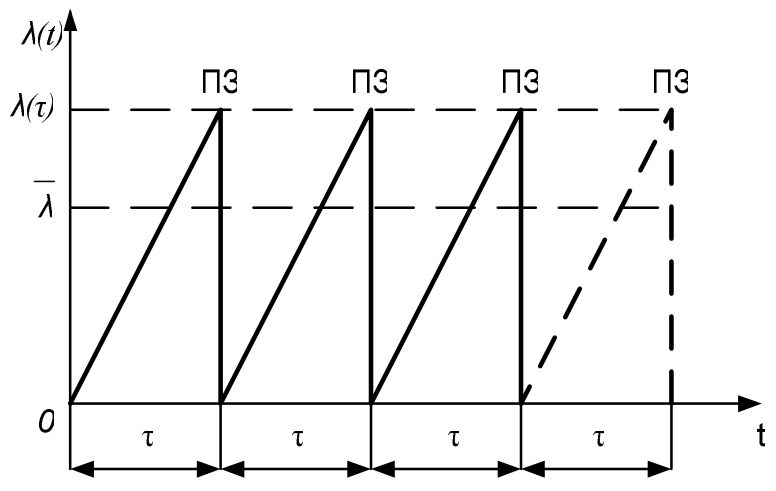

Рис. 1. Изменение интенсивности отказов при заменах ТУ

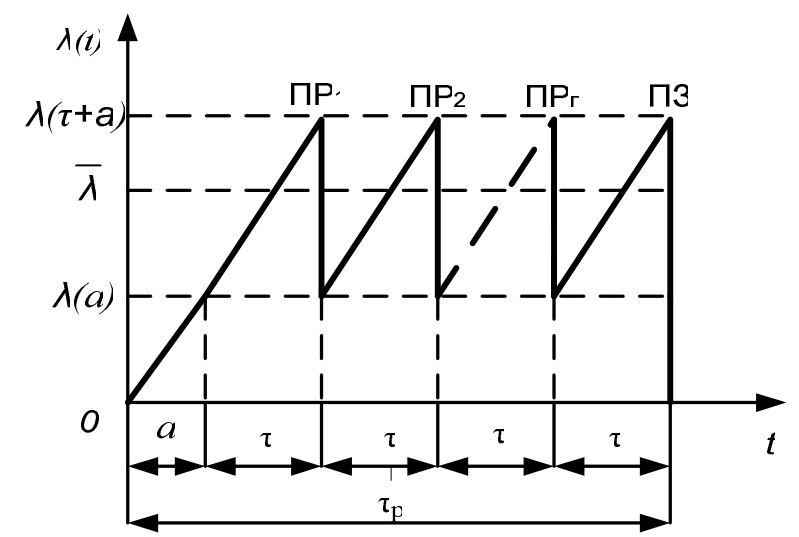

Рис. 2. Изменение интенсивности отказов при ремонтах и замене ТУ

Средняя интенсивность отказов $\bar{\lambda}$, отмеченная на рис. 2 пунктирной линией, на интервале $0-\tau_{p}$ определяется из выражения

$\bar{\lambda}=\tau_{\mathrm{p}}^{-1} \int_{0}^{\tau_{\mathrm{p}}} \lambda(t) d t$

где $\int_{0}^{\tau_{p}} \lambda(t) d t=\int_{0}^{a} \lambda(t) d t+(n+1) \int_{a}^{\tau+a} \lambda(t) d t=$

$=n \ln P(a)-(\mathrm{n}-1) \ln P(\tau+a)$;

$P(a)$ и $P(\tau+a)$ - вероятность безотказной работы соответственно при наработке $a$ и $\tau+a$. 
Подставив значение $\int_{0}^{\tau_{\mathrm{p}}} \lambda(t) d t$, в выражение $(2)$, и учитывая, что $\tau_{p}=a+(n+1) \tau$ (см. рис. 2$)$, получим

$\bar{\lambda}=[n \ln P(a)-(n+1) \ln P(\tau+a)] /[a+(n+1) \tau]$,

Рассмотрим два частных случая:

при $n=0$, когда проводятся только замены, и $a=0$, из (3) получим уравнение (1);

при $n \rightarrow \infty$, когда проводятся только ремонты, после раскрытия неопределенности получим

$$
\bar{\lambda}=[\ln P(a)-\ln P(\tau+a)] \tau^{-1}
$$

В случае, когда отказы ТУ описываются распределением Вейбулла, выражение (3) имеет вид

$\bar{\lambda}=\left[(n+1)((\tau+a) k b / T)^{b}-n(a k b / T)^{b}\right] /[a+(n+1) \tau]$,

где $T$ - наработка на отказ;

$b$ - параметр формы распределения Вейбулла;

$k_{b}=\Gamma(1+1 / b)$, здесь $\Gamma$ - гамма-функция.

C помощью выражения (5) проведены исследования зависимости средней интенсивности отказов от количества и периодичности ПР, глубины восстановления безотказности и параметра формы распределения Вейбулла. Установлено, что с уменьшением количества ПР до замены ТУ значение $\bar{\lambda}$ снижается. Например, в случае распределения Вейбулла при $b=4, T=1000$ час, $a=50$ час, $\tau=200$ час имеем:

$$
\begin{aligned}
& \text { при } n \rightarrow \infty-\bar{\lambda}=0,131 \times 10^{-4} 1 / \text { час; } \\
& \text { при } n=3 \quad \text { - } \quad \bar{\lambda}=0,124 \times 10^{-4} 1 / \text { час; } \\
& \text { при } n=0 \quad-\quad \bar{\lambda}=0,054 \times 10^{-4} 1 / \text { час. }
\end{aligned}
$$

При уменьшении периодичности ПР значение $\bar{\lambda}$ снижается. Например, в случае распределения Вейбулла при $b=4, T=1000$ час, $a=50$ час, $n=3$, получаем:

$$
\begin{aligned}
& \text { при } \tau=200 \text { час - } \bar{\lambda}=0,124 \times 10^{-4} 1 / \text { час, } \\
& \text { а при } \tau=100 \text { час - } \bar{\lambda}=0,030 \times 10^{-4} 1 / \text { час. }
\end{aligned}
$$

При уменьшении параметра $a$ (увеличении глубины восстановления безотказности) значение $\bar{\lambda}$ снижается, а при $a=0$ значение $\bar{\lambda}$ определяется только периодичностью ПЗ. Например, в случае распределения Вейбулла при $b=2, \quad T=1000$ час, $\tau=200$ час, $n=3$ имеем:

$$
\begin{aligned}
& \text { при } a=100 \text { час }-\bar{\lambda}=0,288 \times 10^{-3} 1 / \text { час, } \\
& \text { а при } a=50 \text { час }-\bar{\lambda}=0,224 \times 10^{-3} 1 / \text { час. }
\end{aligned}
$$

Таким образом, уменьшая периодичность или количество ремонтов до замены ТУ или увеличивая глубину восстановления безотказности (путем расширения объема работ по замене или восстановлению составных частей ТУ при проведении ПР), можно обеспечить практически любое заданное значение средней интенсивности отказов технических устройств.

С уменьшением коэффициента вариации $V$ (с ростом параметра $b$ ) значение $\bar{\lambda}$ снижается. Например, в случае распределения Вейбулла при $T=1000$ час, $a=50$ час, $\tau=200$ час, $n=1$ имеем:

при $b=2-\bar{\lambda}=0,214 \times 10^{-3} 1 /$ час,

а при $b=4-\bar{\lambda}=0,117 \times 10^{-3} 1 /$ час.

Это означает, что для технических устройств с явно выраженными процессами старения и износа повышается эффективность проведения предупредительного ремонта.

\section{3. Наработка на отказ ремонтируемых устройств}

Сначала необходимо предложить выражения для определения средней наработки на отказ (ННО) ремонтируемых устройств с учетом глубины восстановления безотказности. Затем исследовать влияние периодичности ремонтов и глубины восстановления безотказности на значения ННО для разных распределений, используемых для описания процессов старения и износа технических устройств. Средняя наработка на отказ ТУ при проведении ПЗ определяется согласно [13] как

$T(\tau)=\int_{0}^{\tau} P(t) d t /(1-P(\tau))$

где $\tau$ - периодичность ПЗ;

$P(t)$ - вероятность безотказной работы за период времени $t$.

Выражение (6) справедливо для случая, когда полностью восстанавливается безотказность ТУ. Это означает, что “возраст” ТУ после ПЗ как бы “возвращается” в нуль. На практике ТУ, как правило, не заменяются, а ремонтируются. При этом не происходит полное восстановление их первоначальной безотказности.

Вероятность безотказной работы (ВБР) при проведении ПР с периодичностью $\tau$ и глубиной восстановления $\alpha$ определяется согласно [1] как условная ВБР $P(\tau / a)$ ТУ, имеющего возраст $\alpha$, из выражения

$P(\tau / \alpha)=P(\tau+\alpha) / P(\alpha)$

Для стареющих ТУ при $\tau>0$ значение $P(\tau / \alpha)$ убывает по $\alpha$. Это означает, что чем с меньшей 
глубиной выполняется ПР, тем ниже значение ВБР ТУ. Подставив в выражение (6) вместо $P(t)$ и $P(\tau)$ значения $P(t / \alpha)$ и $P(\tau / \alpha)$ из (7), получим уравнение для определения ННО ТУ при проведении ПР $T(\tau / \alpha)$ вида

$$
T(\tau / \alpha)=\int_{\alpha}^{\tau+\alpha} P(t) d t /(P(\alpha)-P(\tau+\alpha)) .
$$

Если проводятся ПЗ, то из выражения (8), как частный случай при $\alpha=0$, получим уравнение (6). Для удобства проведения расчетов и анализа полученных результатов приведем выражение (8) к безразмерному виду

$T_{*}=T(\tau / \alpha) / T=\int_{\tau}^{x+\tau} P(u) d u /(P(\alpha)-P(x+\alpha))$,

где $T_{*}$ - относительная ННО ТУ при проведении ПР;

$T$ - ННО ТУ, когда ПР не проводятся;

$x=\tau / T ; \quad \alpha=a / T ; \quad u=t / T-\quad$ соответственно периодичность ПР, глубина восстановления безотказности и время в единицах ННО.

При $x=0$ из выражения (9) после раскрытия неопределенности получим $T_{*}=1 / \lambda(\alpha)$. Это означает, что ННО при проведении ПР не может быть увеличена более, чем на величину, обратную интенсивности отказов в точке $\alpha$, оценивающей глубину восстановления безотказности ТУ. При проведении ПЗ, когда $\alpha=0$, получим $T_{*}=1 / \lambda(0)$. Если $\lambda(0)=0$, то $T_{*}^{*} \rightarrow \infty$. Это означает, что в этом случае при $x \rightarrow 0$ может быть обеспечена практически безотказная работа ТУ.

Известно, что ПЗ или ПР повышают безотказность, если ТУ являются стареющими изделиями, то есть интенсивность отказов у которых возрастает во времени. Рассмотрим два предельных случая: 1) процессы старения в ТУ отсутствуют, что соответствует случаю экспоненциального распределения с $V=1$; 2) ННО ТУ является детерминированной величиной, что соответствует случаю вырожденного распределения с $V=0$.

В случае экспоненциального распределения, когда $P(u)=\exp (-u)$, из выражения (9) получим $T_{*}=1$. Это означает, что ННО не изменяется и проведение ПР нестареющих ТУ нецелесообразно. В случае вырожденного распределения, когда $P(u)=1$ при $u \leq 1$ и $P(u)=0$ при $u>0$, из выражения (9) получим $T_{*} \rightarrow \infty$. Это означает, что при проведении ПЗ с периодичностью $x \leq 1$ обеспечивается абсолютно безотказная работа ТУ.

В случае распределения косинуса, когда $P(u)=\cos u$, a $V=0,376$ [2], выражение (9) имеет вид

$$
T_{*}^{*}=(\sin (x+\alpha)-\sin \alpha)(\cos \alpha-\cos (x+\alpha))^{-1},
$$

причем при $x=0$ после раскрытия неопределенности получим $T_{*}=\operatorname{ctg} \alpha$.

При проведении П3, когда $\alpha=0$, из выражения (10) получим

$$
T_{*}=\sin x(1-\cos x)^{-1}
$$

Зависимости $T_{*}$ от $x$ при различных значениях $\alpha$, построенные с использованием уравнения (10), представлены на рис. 3, на котором горизонтальной пунктирной линией показано значение $T_{*}=1$, когда ремонт не проводится. Из рисунка видно следующее. Во-первых, наработка на отказ ТУ существенно зависит от глубины восстановления безотказности. Например, при периодичности ПР $x=0,2$ и уменьшении глубины восстановления безотказности, то есть при увеличении $\alpha$ от нуля до 0,4 относительная ННО ТУ снижается с 9,97 до 1,83 , то есть в 5,5 раза.

Во-вторых, при $\alpha>0$, начиная с некоторого значения периодичности ПР, которое назовем граничным $x_{\Gamma}$ (см. вертикальные пунктирные линии на рис. 3), значение $T_{*}$ становится менее единицы. Это означает, что проведение ремонта ухудшает безотказность ТУ. Выражение для определения $x_{\Gamma}$ найдем из уравнения (10) при условии, что $T_{*}=1$ :

$x_{\Gamma}=\pi / 2-2 \alpha$.

Аналогично при заданном значении $x$ определяется граничное значение глубины восстановления безотказности $\alpha_{\Gamma}$ :

$\alpha_{\Gamma}=\pi / 4-x / 2$.

Предельное значение глубины восстановления безотказности $\alpha_{\Pi}$, когда при любом значении периодичности ПР $T_{*} \leq 1$, найдем из условия $\lambda\left(\alpha_{\Pi}\right)=1$ как $\alpha_{\Pi}=\operatorname{arctg} 1=\pi / 4 \approx 0,786$. 


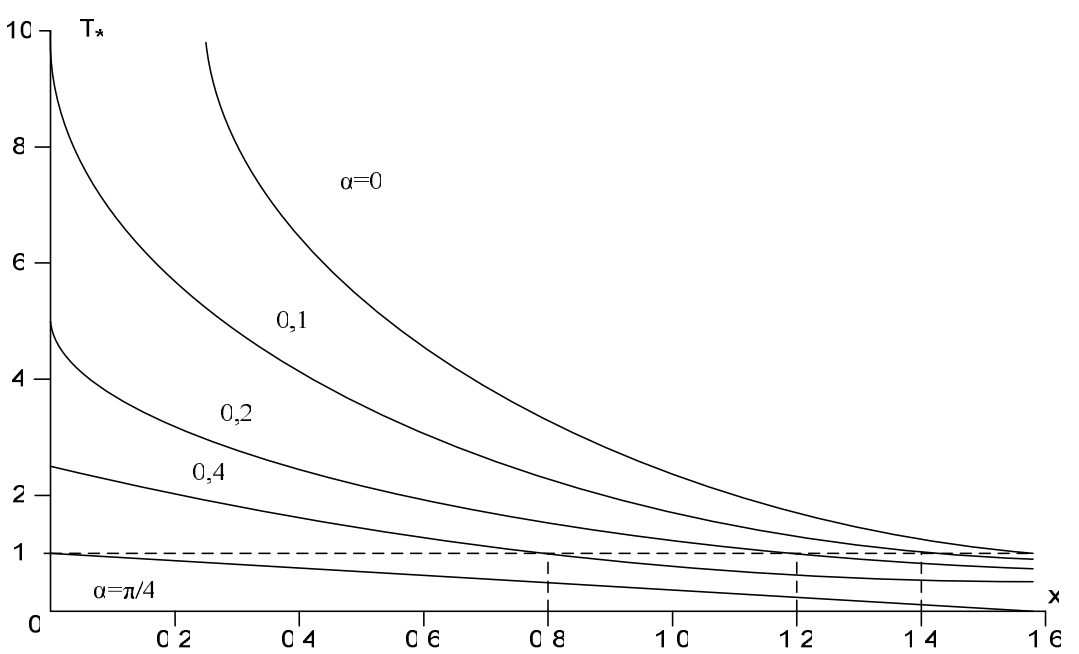

Рис. 3. Зависимости наработки на отказ при распределении косинуса от периодичности предупредительного ремонта и глубины восстановления безотказности

На рис. 4 представлена построенная с использованием уравнения (11) зависимость $\alpha_{\Gamma}$ от $x$ (см. прямая линия 1). Причем, если точка пересечения значения $\alpha_{\Gamma}$ и $x$ лежит выше прямой 1 , то проведение ремонта при такой периодичности и глубине восстановления безотказности ТУ нецелесообразно. В этом случае необходимо или уменьшить периодичность ремонта, или увеличить глубину восстановления безотказности (путем расширения объема работ по замене или восстановлению составных частей ТУ) до таких значений, чтобы точка пересечения $\alpha_{\Gamma}$ и $x$ лежала ниже прямой 1 .

В случае гамма-распределения, когда $P(u)=\exp (-m u) \sum_{0}^{m-1} \frac{(m u)^{i}}{i !}[1]$, при $m=2(V=707)$ из выражения (9) получим

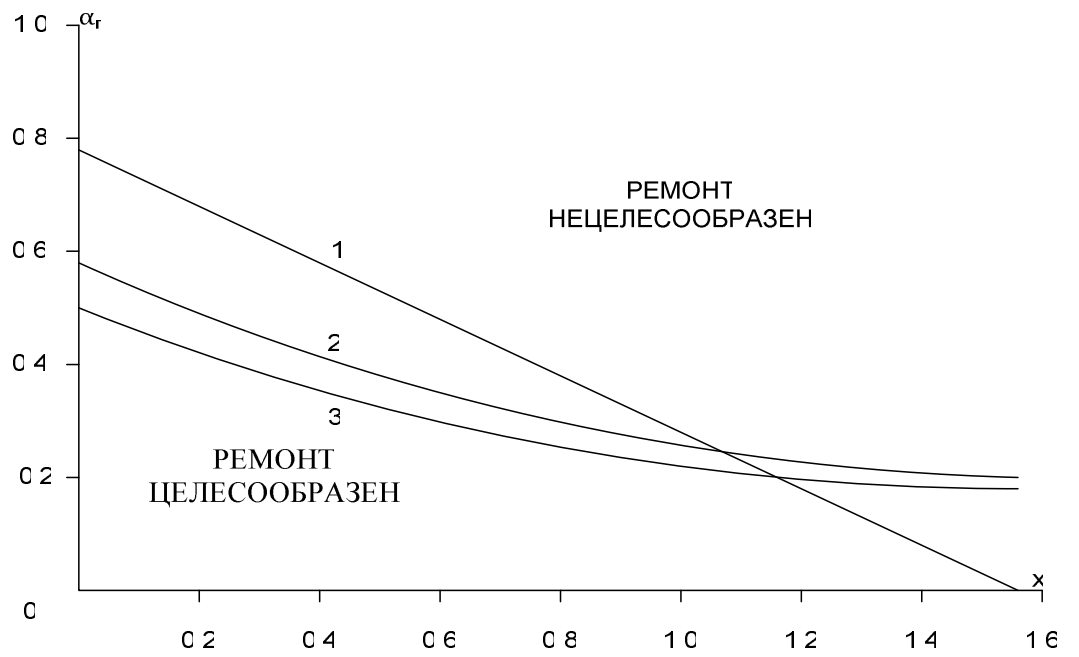

Рис. 4. Зависимости граничных значений глубины восстановления безотказности от периодичности предупредительного ремонта

$$
T_{*}=\frac{(1+\alpha) \exp (-2 \alpha)-(1+x+\alpha) \exp (-2(x+\alpha))}{(1+2 \alpha) \exp (-2 \alpha)-(1+2 x+2 \alpha) \exp (-2(x+\alpha))}
$$

Уравнение для определения $\alpha_{\Gamma}$ найдем из выражения (12) при условии, что $T_{*}=1$ :

$\alpha_{\Gamma}=\operatorname{xexp}(-2 x)(1-\exp (-2 x))^{-1}$

Учитывая, что при $m=2 \lambda(u)=4 u(1+2 u)^{-1}$, предельное значение глубины восстановления безотказности найдем из условия $\lambda\left(\alpha_{\Pi}\right)=1$ как $\alpha_{\Pi}=0,5$. Зависимость $\alpha_{\Gamma}$ от $x$, построенная с использованием уравнения (13), представлена на рис. 4 (кривая 3). 
В случае гамма-распределения при $m=4(V=0,5)$ из выражения (9) получим приближенное решение численным методом $\alpha_{\text {п }} \approx 0,567$. Из полученных результатов видно, что с ростом параметра формы гамма-распределения, то есть с уменьшением коэффициента вариации, возрастает предельное значение глубины восстановления безотказности при проведении предупредительного ремонта. Граничные значения глубины восстановления безотказности в случае гамма-распределения при $m=4$ определены как абсциссы точек пересечения кривых $T_{*}(x)$, построенных с использованием выражения (9), с прямой $T_{*}=1$.

Зависимость $\alpha_{\Gamma}$ от $x$ представлена на рис. 4 (кривая 2). Из сравнения кривых 2 и 3, представленных на рис. 4 , видно, что с уменьшением коэффициента вариации граничные значения глубины восстановления безотказности увеличиваются. Это означает, что для технических устройств, у которых процессы старения выражены более явно, расширяется диапазон целесообразности проведения предупредительного ремонта.

\begin{tabular}{l}
\hline Выводы \\
\hline 1. Для определения
\end{tabular}

2. Значение средней интенсивности отказов с уменьшением периодичности и количества ремонтов до замены технических устройств снижается, а с уменьшением глубины восстановления безотказности возрастает.

3. Варьируя глубину восстановления безотказности или периодичность и количество предупредительных ремонтов за срок службы, можно обеспечить практически любое заданное значение средней интенсивности отказов технических устройств.

4. Наработка на отказ технических устройств существенно зависит от глубины восстановления безотказности при проведении их предупредительного ремонта. Существуют граничные значения периодичности и глубины восстановления безотказности, при которых проведение предупредительных ремонтов нецелесообразно, так как наработка на отказ технических устройств в этих случаях становится ниже, чем при отсутствии такого ремонта.

5. Существуют предельные значения глубины восстановления безотказности, при которых проведение предупредительных ремонтов с любой периодичностью снижает наработку на отказ технических устройств и проведение таких ремонтов нецелесообразно.

6. Полученные результаты показывают, что в условиях эксплуатации за счет изменения периодичности проведения предупредительных ремонтов и глубины восстановления безотказности можно обеспечить требуемое значение наработки на отказ технических устройств.

7. Для технических устройств с явно выраженными процессами старения и износа эффективность проведения предупредительного ремонта повышается. В этом случае при прочих равных условиях у технических устройств, имеющих распределение наработки на отказ с меньшим значением коэффициента вариации, средняя интенсивность отказов ниже.

\section{Список использованных источников}

1. Барлоу, Р. Статическая теория надежности и испытания на безотказность [Текст] / Р. Барлоу, Ф. Прошан. - М.: Наука, 1984. - 328 с.

2. Володарский, В. А. Аппроксимация распределения вероятности безотказной работы функцией косинуса [Текст] / В.А. Володарский // Надежность и контроль качества. - 1988. - №8. - С. 18-22.

3. Beichelt T., Fisher K. General failure model applied to preventive maintenance policies // IEEE Transactions on reliability. - 1980. - Vol. R-29, № 1. - P. 39-41.

4. Разгильдеев, Г. И. Математическая модель замены электрооборудования с учетом неравноценности отказов [Текст] / Г.И. Разгильдеев, А.Г. Захарова // Известия вузов. Энергетика. - 1981. - №9. C. $79-84$.

5. Helvik, B. Periodic maintenance on the effect of imperfectness // $10^{\text {th }}$ Int. Symp. Fault Tolerant Comput. - Kyoto, Oct. 1-3, 1980. - P. 204-206.

6. Murthy D.N.P., Ngugen D.G. Optimal age - policy with imperfect preventive maintenance// IEEE Transactions on reliability. - 1981. - Vol. R-30, № 1. - P. 80-81.

7. Рудь, Ю.С. Оптимизация технического обслуживания технологического оборудования горно-обогатительных комбинатов [Текст] / Ю.С. Рудь // Известия вузов. Горный журнал. 1980. - № 12. - С. 47-51.

8. Моломин, В. П. Управление надежностью авиационной техники [Текст] / В.П. Моломин. М.: Машиностроение, 1981. - 200 с.

9. Malik M. A. K. Reliable preventive maintenance scheduling // AIIE Trans. - 1979. - Vol. 11. P. 221-228.

10. Черепанов, С. С. Техническое обслуживание и ремонт машин в сельском хозяйстве [Текст] / С.С. Черепанов. - М.: Колос, 1979. - 288 с.

11. Володарский, В. А. Об оптимизации предупредительных замен и ремонтов технических 

C. 21-24.

12. Эндрени, Дж. Моделирование при расчетах надежности в электроэнергетических системах [Текст] / Дж. Эндрени. - М.: Энергоатомиздат, 1983. $-435 \mathrm{c}$.

13. Барлоу, Р. Математическая теория надежности [Текст] / Р. Барлоу, Ф. Прошан. - М.: Сов. радио, 1969. - 488 c.

Volodarsky V.A. About reliability of technical devices, undergone repair. Article purpose - to offer and investigate expressions for definition of average failure rate and a time between failures of the repaired technical devices, the non-failure operation restitutions differing in depth.Value of average failure rate with decrease of frequency and number of repairs decreases to replacement of technical devices, and with decrease of depth of restitution of non-failure operation increases. Varying depth of restitution of non-failure operation or frequency and the number of preventive repairs for endurance, it is possible to provide practically any preset value of average failure rate of technical devices. The time between failures of technical devices significantly depends on depth of restitution of non-failure operation when carrying out their preventive repair. There are boundary values of frequency and depth of restitution of non-failure operation at which carrying out preventive repairs is inexpedient as the time between failures of technical devices in these cases becomes lower, than in the absence of such repair. There are limiting values of depth of restitution of non-failure operation at which carrying out preventive repairs with any frequency reduces a time between failures of technical devices and carrying out such repairs is inexpedient. The received results show that under operating conditions due to change of frequency of carrying out preventive repairs and depth of restitution of non-failure operation it is possible to provide a desired value of a time between failures of technical devices. For technical devices, with obviously expressed processes of an aging and wear, effectiveness of carrying out preventive repair increases. In this case with other things being equal at the technical devices having distribution of a time between failures with smaller value of a variation factor, average failure rate is lower.

Keywords: failure rate, time between failures, non-failure operation, restitution depth, repair, replacement, frequency.

Надійшла 28.12.2016 p.

Volodarsky Vladislav A., PhD, senior researcher, associate professor "Krasnoyarsk Institute of Railway Transport», Krasnoyarsk, Russia. E-mail: volodarsky.vladislav@yandex.ru 